\title{
Acute Arterial Occlusion Following Primary Total Knee Arthroplasty
}

\author{
Bong-Ju Park, MD, Hong-Man Cho, MD, Ki-Yong An, MD, and Hyun-Ju Lee, MD \\ Department of Orthopedic Surgery, Gwangju Veterans Hospital, Gwangju, Korea
}

Acute arterial occlusion is a rare complication following total knee arthroplasty (TKA). This is a report of a case of acute femoral artery occlusion and its sequelae following TKA in a patient with a history of atrial fibrillation. Arterial circulation of the lower limb could not be restored by thrombectomy treatments, and above-knee amputation had to be carried out.

Keywords: Knee, Arthroplasty, Artery, Occlusion, Atrial fibrillation

As the general population ages, the number of patients with degenerative knees also continues to increase ${ }^{1)}$. In addition, more patients of advanced age are choosing surgical treatment to improve their quality of life. Total knee arthroplasty (TKA) is the most cost-effective and safest procedure for restoring function to an arthritic knee joint ${ }^{2}$. However, despite its safety, TKA may have unsatisfactory outcome associated with immediate or shortterm complications after surgery in a population with other concomitant diseases. Perioperative complications include blood loss, infection, early hemorrhage and wound breakdown, intraoperative fractures, and anesthetic problems as well as cardiovascular, respiratory, renal, electrolyte, and other medical problems ${ }^{3)}$. Acute arterial occlusion is rarely associated with TKA. A rare but serious complication is occlusion of the artery, the incidence of which is stated to be $0.033 \%{ }^{4)}$ in large patient populations. Here, we describe a case of acute arterial occlusion after TKA associated with atrial fibrillation (AF). The case presented in this report

Received April 17, 2017; Revised (1st) June 8, 2017; (2nd) July 5, 2017; Accepted July 10, 2017

Correspondence to: Hong-Man Cho, MD

Department of Orthopedic Surgery, Gwangju Veterans Hospital, 99

Cheomdanwolbong-ro, Gwangsan-gu, Gwangju 62284, Korea

Tel: +82-62-602-6162, Fax: +82-62-602-6936

E-mail: chm1228@hanmail.net

This is an Open Access article distributed under the terms of the Creative Commons Attribution Non-Commercial License (http://creativecommons.org/licenses/by-nc/4.0/) which permits unrestricted non-commercial use, distribution, and reproduction in any medium, provided the original work is properly cited. is different from that in previous studies in that thrombotic occlusion occurred even after adequate prophylaxis following knee arthroplasty in a patient who had AF.

\section{Case Report}

An 83-year-old male patient had osteoarthritis for 7 years in the right knee, with no flexion contracture or highly varus and valgus deformity. Because of extreme pain, the patient underwent magnetic resonance imaging, which showed lesions in the medial femoral condyle cartilage in the weight-bearing region; the same findings were confirmed during the TKA operation (Fig. 1). He had previously undergone left primary TKA for incapacitating knee pain recalcitrant to conservative therapy at our hospital 25 months ago. We considered salvage procedures such as cartilage restoration procedure or high tibial osteotomy considering the size of the knee joint lesion and combined medical disease with age. However, we planned TKA since the patient complained of severe pain, and satisfaction was high on the contralateral side after TKA. He had been taking warfarin for paroxysmal AF. He had a smoking history of 1.5 packs per day for 32 years and cholecystectomy 2 years ago, but he had no specific medical history or history of peripheral vascular disease, being bed-ridden for a long period, or glucocorticoid usage. The patient had normal femoral, popliteal, and ankle pulses bilaterally on palpation. The ankle brachial index (ABI; right, 1.06; left, 1.04) and echocardiography showed no specific clinical manifestation. After 3 

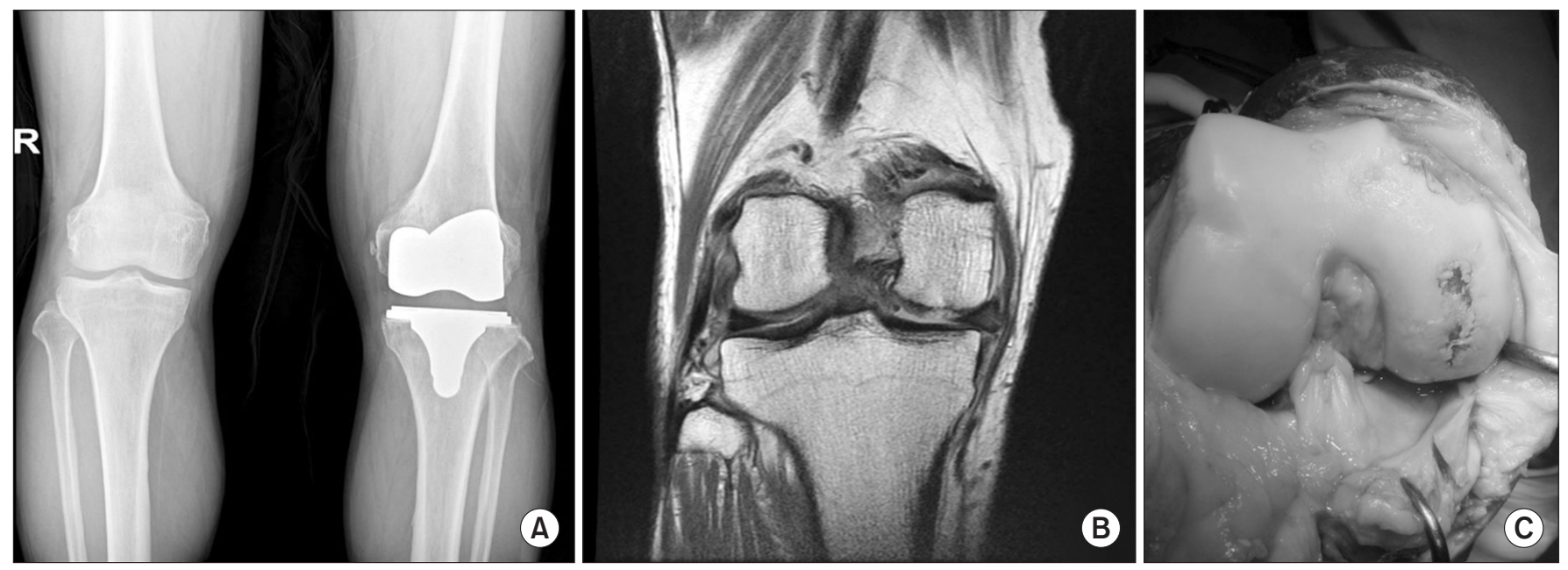

Fig. 1. (A) Radiograph showing proximal tibial vara in the right knee and previous total knee arthroplasty status of the left knee. (B) T1-weighted coronal magnetic resonance imaging scan showing degeneration of the medial meniscus, loss of articular cartilage in the medial femoral condyle, and tiny marginal osteophytes in the right knee joint. (C) Removal of the right knee joint capsule with cartilage loss in the medial femur.

days of warfarin hold for perioperative bridging anticoagulation therapy, the patient's international normalized ratio (INR) was 1.72. Enoxaparine sodium ( $40 \mathrm{mg}$ Cnoxane; YooYoung Pharm co., Seoul, Korea) was administered to the patient. He underwent TKA under spinal anesthesia. TKA was initiated with a tourniquet inflated to a pressure of $300 \mathrm{mmHg}$, and the anterior parapatellar approach was used. The total blood loss for the procedure was $350 \mathrm{~mL}$, which was measured by weighing the gauze plus the suction drain amount without the irrigating solution. The tourniquet time lasted for 96 minutes. No intraoperative complications were noted during the surgical procedure. Compressive dressing was applied on the lower limb after the operation. After surgery, the patient was transferred to the recovery room. The patient's motor and sensory functions were not checked in the recovery room due to spinal anesthesia, but the patient's lower limb was warm and the pulsation of the dorsalis pedis artery was intact. After about 20 minutes in the recovery room, the patient's mental state declined and he became drowsy, his respiration decreased, and his blood pressure decreased to $80 / 40 \mathrm{mmHg}$; thus, he was transferred to the intensive care unit. Computed tomography (CT) images showed no specific lesions in the brain and lungs. Supportive therapy was administered, and the patient's vital signs recovered to normal range on postoperative day 1 . However, on postoperative day 1 , after waking up, the patient showed an unstable mental status without awareness of places or persons and signs of delirium; he was trembling and tried to get out of bed. He was given a sedative, which did not provide much improvement, and restraint bands were used to limit the patient's hand and foot movements. On postoperative day 2, the drain tube was removed because the Hemovac drain amount decreased to 60 $\mathrm{mL}$. To remove the drain, the compressive dressing was removed for the first time after surgery. However, the pulse and temperature of the lower limb were not checked, which should have also been checked on postoperative day 1 . We should have classified him as a high risk patient for thrombotic events, but which we did not. If we had examined more carefully, arterial occlusion could have been found earlier. On postoperative day 2, Cnoxane $40 \mathrm{mg}$ and warfarin were administered because the patient's INR increased to 1.83. An air pressure device was used for mechanical prophylaxis to prevent deep vein thrombosis after the removal of the compressive dressing. On postoperative day 3 , the dosage of warfarin was the same as that used before the surgery. On the afternoon of postoperative day 2 , when the compressive dressing was removed for dressing change, there was notable necrosis of the foot on gross examination. No pulsation of the dorsalis pedis artery was noted during palpation or capillary filling of the toenail. Emergency lower-extremity CT angiography showed total occlusion of the right femoral artery (Fig. 2). After emergency consultation with the cardiovascular department, angiography was performed, which showed total embolic occlusion of the right femoral artery. Fogarty thrombectomy was attempted via the right inguinal site; however, the reperfusion of the popliteal and anterior and posterior tibial arteries was not satisfactory (Fig. 3). No back bleeding was noted, which means that there is no bleeding through the catheter where it is approached below the occluded artery. Open thrombectomy was performed when the occlusion site was not completely resolved, but flow maintenance was observed on completion angiography, thus precluding the 

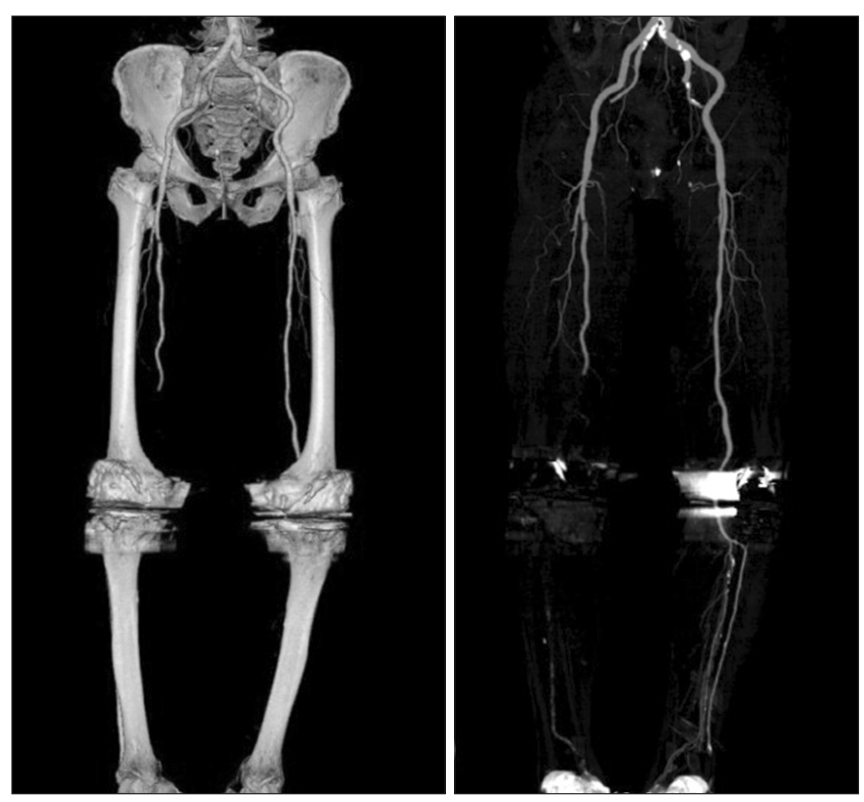

Fig. 2. Computed tomography angiography showing superficial femoral artery occlusion in the right limb.

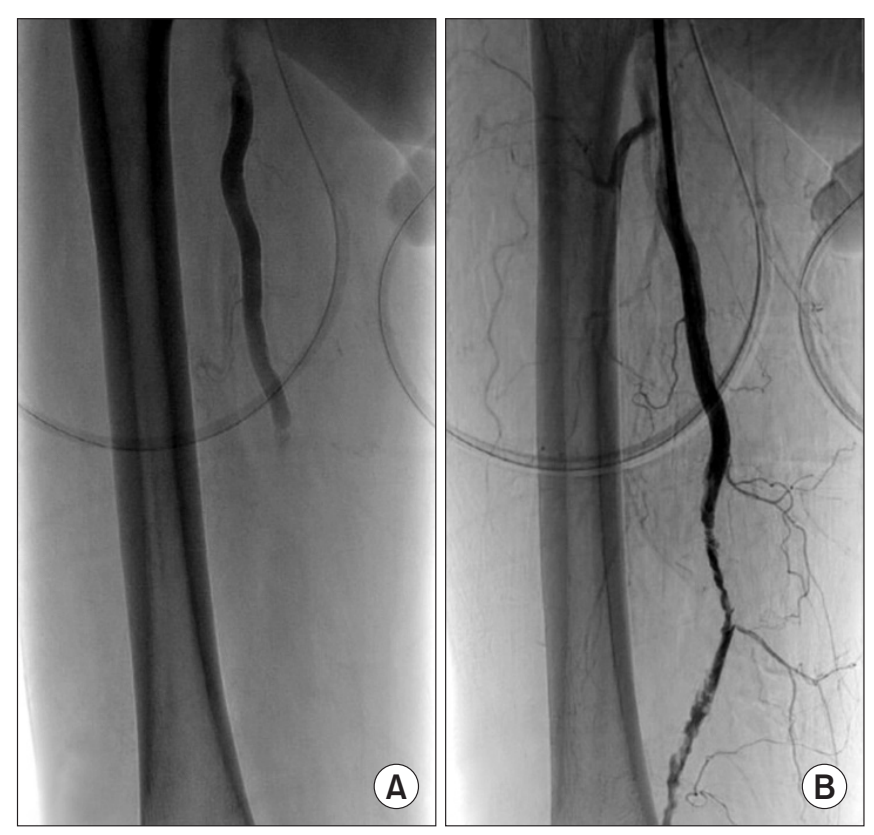

Fig. 3. (A) Arteriogram showing right superficial femoral artery occlusion. (B) Arteriogram showing the reperfusion of the superficial femoral, popliteal, and anterior and posterior tibial arteries after Fogarty thrombectomy.

need for additional open thrombectomy. However, upon evaluation, we were dissatisfied because the flow was insufficient. On postoperative day 4 , sepsis combined with rhabdomyolysis was suspected. The patient was drowsy, with decreased blood pressure $(70 / 50 \mathrm{mmHg})$, and the necrosis below the knee deteriorat-

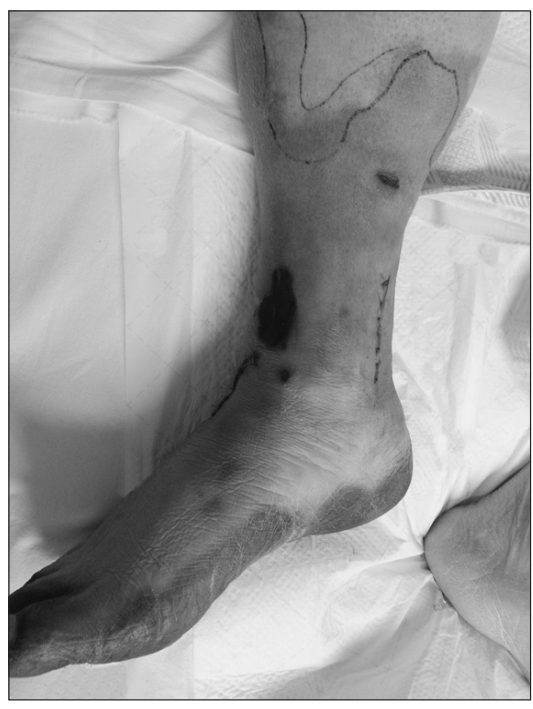

Fig. 4. Photograph showing necrosis of the lower leg on postoperative day 7.

ed rapidly (Fig. 4). On postoperative day 17 , above-knee amputation was performed (Fig. 5). After amputation, the vital signs of the patient stabilized. He was discharged, using a wheelchair for ambulation. The patient has been monitored for 6 months after amputation.

\section{Discussion}

Arterial complications after TKA are relatively uncommon. Although infrequent, the arterial complications after TKA are heterogeneous; the most frequently reported ones are arterial thrombosis, arterial transection, arteriovenous fistula, and aneurysm formation ${ }^{5}$. The reported incidence of complications after TKA including arterial occlusion, arteriovenous fistula, arterial aneurysm, and arterial severance ranged from $0.03 \%$ to $0.17 \%{ }^{6}$. Nonetheless, the infrequency of these complications may make the diagnosis and treatment extremely challenging to surgeons, and limb-threatening ischemia may subsequently occur ${ }^{7}$. Two possible causes of necrosis were considered in this case. The first is the patient's AF. AF is a very common type of arrhythmia, and its prevalence increases significantly with age. This can lead to an increase in blood loss and thrombotic events such as cerebrovascular accident during the perioperative surgical period. AF patients need long-term (usually life-long) anticoagulation therapy to decrease the risk of a thrombotic event ${ }^{8}$. We presented a case of arterial occlusion due to thrombosis despite the use of perioperative bridging anticoagulation therapy (warfarin and enoxaparine). 

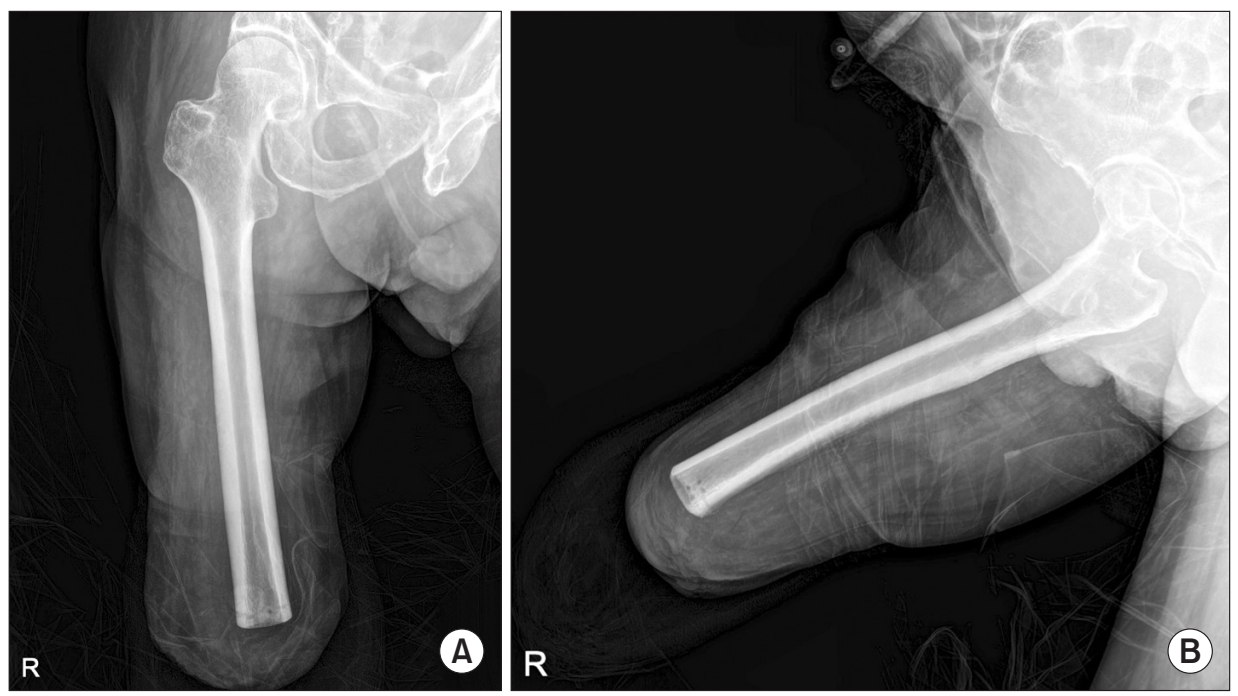

Fig. 5. Radiographs of the right femur after above-knee amputation: anteroposterior (A) and lateral (B).

A higher risk of cardiocerebral events, including acute myocardial infarction and cerebral stroke, has been reported in AF. In addition, orthopedic surgery can significantly increase this risk on account of the hypercoagulability status, elevated sympathetic activation, and higher level of catecholamine ${ }^{9)}$, and patients with a history of AF were more likely to develop thrombotic events after orthopedic surgery ${ }^{10)}$. To the best of our knowledge, only a few studies have assessed the impact of AF on the outcomes after $\mathrm{TKA}^{10)}$. In addition to the complication experienced in this present case, which is very rare, given the implementation of appropriate perioperative bridging anticoagulation therapy, the lower limb arterial condition of the patient was overlooked. Close observation of blood circulation in the lower extremity should be done regularly in patients with high thrombotic risk such as AF. Although the screening tests (palpation and ABI test) of the lower extremity arterial circulations showed no preoperative abnormality, a more precise examination of the lower limb artery of the patient was not performed before the operation. We suggest that Doppler ultrasound or CT angiography may be useful in preventing such fatal complications in patients at high risk for thrombotic events after orthopedic surgery. Furthermore, using a tourniquet in TKA is controversial, as plaque disruption has been reported after tourniquet use in patients with calcification of the femoral or popliteal artery ${ }^{11)}$. It has been suggested that the mechanical pressure from the tourniquet may traumatize atheromatous vessels, causing fractures and dislodgment of the plaque, especially in old patients ${ }^{11)}$. The vessels of old patients may have calcified and lost their elasticity, and intimal tear and plaque embolization could result from the fixation of the femoral artery by the tourniquet and the stretching of the distal artery during intraoperative manipulation ${ }^{12)}$. Therefore, if calcified blood vessels are noted in preoperative plain radiographs, it is necessary to pay more attention to the use of customary tourniquet.

Acute arterial occlusions are serious and can lead to amputation if inadequately treated. Treatment comprises anticoagulation or surgical intervention, which includes thrombectomy and bypass grafting. Therefore, surgeons should take care to prevent the occurrence of this serious complication and avoid the worst scenario, i.e., amputation. Patients who have acute limb occlusion with severe symptoms such as sensory loss in the toe, rest pain, and moderate motor deficit require emergent surgical revascularization $^{13)}$. However, in this case, we considered the patient's discomfort after surgery due to delirium and missed the appropriate period of treatment by not performing a basic physical examination of the lower extremities on postoperative day 2. Therefore, we suggest careful checking of motor, sensory, and circulation of patients' lower extremity, which is a basic but highly overlooked procedure in patients at high risk for thrombotic events after orthopedic surgery. If acute occlusion of the lower limb is identified, bypass grafting and revascularization ${ }^{6}$ or thrombectomy using an aspiration catheter ${ }^{12)}$ including thrombolytic therapy is necessary. Thrombectomy is the most frequently described treatment, which can lead to complete recovery; however, in patients with extreme necrosis, as in this case, thrombectomy might be futile and ultimately need amputation. In our case, the patient presented with foot coldness, decreased sensation, and paresthesias, thus prompting immediate thrombectomy with a Fogarty catheter for revascularization of acute arterial occlusion, which, however, failed. Calligaro et al. ${ }^{6}$ reported the largest single-center experience with management of acute ischemic complications associated with TKA. They claimed that this complication was best managed by an aggressive protocol including arterial bypass and 
emergency revascularization. However, in their report, arterial thrombectomy was only successful in approximately one-fourth $(5 / 18,28 \%)$ of the patients, and they had no associated intimal damage $^{6)}$. Therefore, thrombectomy could not restore the circulation. For the patients in this case, arterial bypass may be the better choice.

In conclusion, in the case of TKA in a patient with concomitant medical condition and risk of an arterial complication, the patient should be evaluated adequately for the existence of arterial disease before operation, for example, by using ultrasound sonography and enhanced CT. The preoperative evaluation of the patient's arterial condition should be considered to avoid acute arterial occlusive disease. Moreover, the fundamental process of examination of blood circulation in the lower extremity performed periodically and thoroughly after surgery is very important in patients at high risk for thrombotic events.

\section{Conflict of Interest}

No potential conflict of interest relevant to this article was reported.

\section{References}

1. Zhang Y, Jordan JM. Epidemiology of osteoarthritis. Clin Geriatr Med. 2010;26:355-69.

2. Jones CA, Beaupre LA, Johnston DW, Suarez-Almazor ME. Total joint arthroplasties: current concepts of patient outcomes after surgery. Clin Geriatr Med. 2005;21:527-41.

3. Menendez ME, Memtsoudis SG, Opperer M, Boettner F, Gonzalez Della Valle A. A nationwide analysis of risk factors for in-hospital myocardial infarction after total joint arthroplasty. Int Orthop. 2015;39:777-86.
4. Rand JA. Vascular complications of total knee arthroplasty: report of three cases. J Arthroplasty. 1987;2:89-93.

5. Langkamer VG. Local vascular complications after knee replacement: a review with illustrative case reports. Knee. 2001;8:259-64.

6. Calligaro KD, Dougherty MJ, Ryan S, Booth RE. Acute arterial complications associated with total hip and knee arthroplasty. J Vasc Surg. 2003;38:1170-7.

7. Gregory PC, Rogic R, Eddington C. Acute arterial occlusion after total knee arthroplasty. Am J Phys Med Rehabil. 2006; 85:924-6.

8. Schulman S, Hwang HG, Eikelboom JW, Kearon C, Pai M, Delaney J. Loading dose vs. maintenance dose of warfarin for reinitiation after invasive procedures: a randomized trial. J Thromb Haemost. 2014;12:1254-9.

9. Kurtz S, Ong K, Lau E, Mowat F, Halpern M. Projections of primary and revision hip and knee arthroplasty in the United States from 2005 to 2030. J Bone Joint Surg Am. 2007;89: 780-5.

10. Aggarwal VK, Tischler EH, Post ZD, Kane I, Orozco FR, Ong A. Patients with atrial fibrillation undergoing total joint arthroplasty increase hospital burden. J Bone Joint Surg Am. 2013;95:1606-11.

11. Smith DE, McGraw RW, Taylor DC, Masri BA. Arterial complications and total knee arthroplasty. J Am Acad Orthop Surg. 2001;9:253-7.

12. Berger C, Anzbock W, Lange A, Winkler H, Klein G, Engel A. Arterial occlusion after total knee arthroplasty: successful management of an uncommon complication by percutaneous thrombus aspiration. J Arthroplasty. 2002;17:227-9.

13. Sedghi Y, Collins TJ, White CJ. Endovascular management of acute limb ischemia. Vasc Med. 2013;18:307-13. 